RAIRO-Inf. Theor. Appl. 39 (2005) 511-545

DOI: 10.1051/ita:2005028

\title{
ADHESIVE AND QUASIADHESIVE CATEGORIES *
}

\author{
Stephen LaCK ${ }^{1}$ AND PaWę Sobociński ${ }^{2}$
}

\begin{abstract}
We introduce adhesive categories, which are categories with structure ensuring that pushouts along monomorphisms are wellbehaved, as well as quasiadhesive categories which restrict attention to regular monomorphisms. Many examples of graphical structures used in computer science are shown to be examples of adhesive and quasiadhesive categories. Double-pushout graph rewriting generalizes well to rewriting on arbitrary adhesive and quasiadhesive categories.
\end{abstract}

Mathematics Subject Classification. 18A30, 18A35, 18D99, 68Q42, 68Q65.

\section{INTRODUCTION}

Recently there has been renewed interest in reasoning using graphical methods, particularly within the fields of mobility and distributed computing $[15,21]$ as well as applications of semantic techniques in molecular biology [4,6]. Research has also progressed on specific graphical models of computation [20]. As the number of various models grows, it is important to understand the basic underlying principles of computation on graphical structures. Indeed, a solid understanding of the foundations of a general class of models (provided by adhesive categories), together with a collection of general semantic techniques (for example [23]) will provide practitioners and theoreticians alike with a toolbox of standard techniques with

Keywords and phrases. Adhesive categories, quasiadhesive categories, extensive categories, category theory, graph rewriting.

* The first author acknowledges the support of the Australian Research Council. The second author acknowledges the support of BRICS, Basic Research in Computer Science

(www.brics.dk), funded by the Danish National Research Foundation.

${ }^{1}$ School of Quantitative Methods and Mathematical Sciences, University of Western Sydney, Australia.

${ }^{2}$ BRICS, University of Aarhus, Denmark; pawel@brics.dk

(c) EDP Sciences 2005 
which to construct the models, define the semantics and derive proof-methods for reasoning about these.

Category theory provides uniform proofs and constructions across a wide range of models. The usual approach is to find a natural class of categories with the right structure to support the range of constructions particular to the application area. A well-known example is the class of cartesian-closed categories, which provides models for simply typed lambda calculi [19].

In this paper we shall demonstrate that adhesive categories have structure which allows a development of a rich general theory of double-pushout (DPO) rewriting [14]. DPO graph rewriting has been widely studied and the field can be considered relatively mature $[8,13,22]$.

In DPO rewriting, a rewrite rule is given as a span $L \leftarrow K \rightarrow R$. Roughly, the intuition is that $L$ forms the left-hand side of the rewrite rule, $R$ forms the righthand side and $K$, common to both $L$ and $R$, is the sub-structure to be unchanged as the rule is applied. To apply the rule to a structure $C$, one first needs to find a match $L \rightarrow C$ of $L$ within $C$. The rule is then applied by constructing the missing parts ( $E, D$ and arrows) of the following diagram<smiles>[Y]1[Te][Te][Te]1</smiles>

in a way which ensures that the two squares are pushout diagrams. Once such a diagram is constructed we may deduce that $C \longrightarrow D$, that is, $C$ rewrites to $D$.

DPO rewriting is formulated in categorical terms and is therefore portable to structures other than directed graphs. There have been several attempts $[9,11]$ to isolate classes of categories in which one can perform DPO rewriting and in which one can show that such rewriting grammars satisfy useful properties. In particular, several axioms were put forward in [11] in order to prove a local Church-Rosser theorem for such general grammars. Additional axioms were needed to prove a general version of the so-called concurrency theorem [16].

An important general construction which appears in much of the literature on graphical structures in computer science is the pushout construction. Sometimes referred to as generalized union [9], it can often be thought of as the construction of a larger structure from two smaller structures by gluing them together along a shared substructure.

One can think of adhesive categories as categories in which pushouts along monomorphisms are "well-behaved", where the paradigm for behaviour is given by the category of sets. An example of the good behaviour of these pushouts is that they are stable under pullback (the dual notion to pushout, which intuitively can often be thought of as a "generalized intersection"). The idea is analogous to that of extensive categories [3], which have well-behaved coproducts in a similar sense. Since coproducts can be obtained with pushouts and an initial object, and an initial object is "well-behaved" if it is strict, one might expect that adhesive 
categories with a strict initial object would be extensive, and this indeed turns out to be the case.

Various notions of graphical structures used in computer science form adhesive categories. This includes ordinary directed graphs, typed graphs [1] and hypergraphs [11], amongst others. Indeed, it turns out that any elementary topos is adhesive, although this shall be proved elsewhere [18]. The structure of adhesive category allows us to derive useful properties. For instance, the union of two subobjects is calculated as the pushout over their intersection, which corresponds well with the intuition of pushout as generalized union.

The notion of adhesivity is too strong for several relevant examples. These examples motivate the study of quasiadhesive categories. As a topos is adhesive, so a quasitopos is quasiadhesive [18]; a fact which has guided our terminology. Roughly, instead of focusing on the behaviour of pushouts along arbitrary monomorphisms, quasiadhesive categories restrict attention to pushouts along regular monomorphisms. Quasiadhesive categories are shown to encompass several categories of interest to computer scientists, including the category Spec of algebraic specifications and algebraic specification morphisms.

Adhesive categories can be seen as a degenerate quasiadhesive category, namely one where all monomorphisms are regular. While the theory of quasiadhesive categories generalises the theory of adhesive categories, we believe it is nonetheless useful to study the stronger property of adhesivity because of its simplicity; helpful when dealing with examples which do not require the extra generality.

This paper is an expanded version of the extended abstract [17]. It extends this earlier version with full proofs, some new results about adhesive categories and a more complete treatment of the relationship between adhesive categories and HLRcategories. It also introduces the class of quasiadhesive categories, foreshadowed in [17].

We shall consider adhesive grammars and quasiadhesive grammars, which are DPO grammars on, respectively, adhesive and quasiadhesive categories. We prove the local Church-Rosser theorem and the concurrency theorem without the need for extra axioms. We shall also examine how adhesive and quasiadhesive categories fit within the previously conceived general frameworks for rewriting $[9,11]$. Many of the axioms put forward in [11] follow elegantly as lemmas from the axioms of adhesive categories.

Adhesive and quasiadhesive categories, therefore, provide an abstract setting in which it is possible to define a theory of DPO rewriting. They are mathematically elegant and arguably less ad hoc than previous approaches.

Structure of the paper. In Section 1 we recall the definition of extensive categories. The notion of van Kampen (VK) square is given in Section 2. VK squares are central in the definition of adhesive categories which are introduced in Section 3. In Section 4 we state and prove some basic lemmas which hold in any adhesive category. In Section 5 we show that, in adhesive categories, the subobjects of any object form a distributive lattice, with the union of two subobjects constructed as the pushout over their intersection. Quasiadhesive categories are motivated and 
defined in Section 6. We develop double-pushout rewriting theory in adhesive categories in Section 7 and offer a comparison with High-Level Replacement Categories in Section 8. We conclude in Section 9 with directions for future research.

Conventions. Throughout the paper we assume that the reader is familiar with basic concepts of category theory. We adopt the convention in diagrams of usually not writing labels for identities, arrows to terminal objects, arrows from initial objects and (binary) coproduct coprojections. When we do need to name coprojections, we use $i_{1}$ and $i_{2}$ to refer to, respectively, the first and the second coprojection, letting the context determine exactly which coproduct is discussed.

\section{Extensive CATEGORIES}

We shall repeatedly use basic properties of pushouts and pullbacks, and in particular, the following well-known lemma, sometimes referred to as the pasting lemma. Since pushouts and pullbacks are dual, there are two versions of the lemma.

Lemma 1.1. Given a commutative diagram:

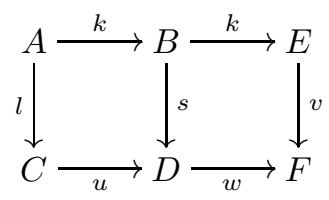

Pullback version - If the right square is a pullback then the left square is a pullback if and only if the whole rectangle is a pullback.

Pushout version - If the left square is a pushout then the right square is a pushout if and only if the whole rectangle is a pushout.

In the remainder of this section we recall briefly the notion of extensive category [3].

Definition 1.2. A category $\mathbf{C}$ is said to be extensive when

(i) it has finite coproducts;

(ii) it has pullbacks along coproduct coprojections;

(iii) given a diagram where the bottom row is a coproduct diagram

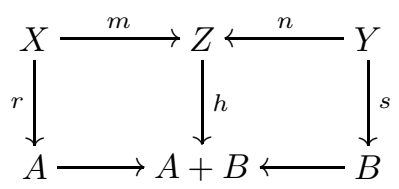

the two squares are pullbacks if and only if the top row is a coproduct.

The third axiom states what we mean when we say that the coproduct $A+B$ is "well-behaved": it includes the fact that coproducts are stable under pullback, and it implies that coproducts are disjoint (the pullback of the coprojections is 
initial) and that initial objects are strict. ${ }^{1}$ It also implies a cancellativity property of coproducts: given an isomorphism $A+B \cong A+C$ compatible with the coprojections, one can construct an isomorphism $B \cong C$. For an object $Z$ of an extensive category, the lattice $\operatorname{Sub}(Z)$ of coproduct summands of $Z$ is a Boolean algebra.

This third axiom is actually equivalent to requiring certain canonical functors to be equivalences of categories (Prop. 1.3, (i)). This is sometimes taken to be the definition of extensive categories; apart from elegance, it has the added advantage of not requiring pullbacks along coprojections.

Proposition 1.3. Given a category $\mathbf{C}$, the following conditions are equivalent for all objects $A$ and $B$ :

(i) the functor $\mathbf{C} / A \times \mathbf{C} / B \rightarrow \mathbf{C} /(A+B)$, which forms the coproducts of morphisms in $\mathbf{C}$, is an equivalence of categories;

(ii) in a commutative diagram

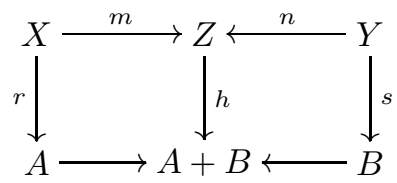

the top row is a coproduct diagram if and only if the squares are pullbacks;

(iii) (when $\mathbf{C}$ has pullbacks along coproduct coprojections) the functor

$$
\mathbf{C} /(A+B) \rightarrow \mathbf{C} / A \times \mathbf{C} / B
$$

given by pullback along the coprojections $A \rightarrow A+B$ and $B \rightarrow A+B$ is an equivalence of categories.

The following is a simple lemma about pullbacks in extensive categories; it states that pullbacks commute with coproducts.

Lemma 1.4. Suppose that $\mathbf{C}$ is an extensive category and that diagrams (i) and (ii) below are pullbacks in $\mathbf{C}$.

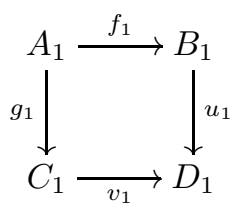

(i)

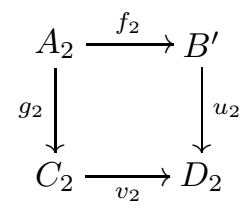

(ii)

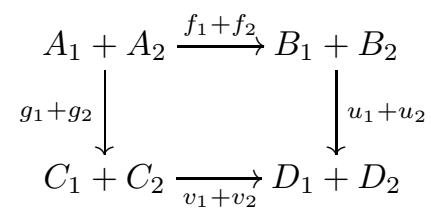

(iii)

Then diagram (iii) is a pullback in $\mathbf{C}$.

\footnotetext{
${ }^{1}$ An initial object is said to be strict precisely when any arrow with the initial object as its codomain is an isomorphism.
} 
Proof. Diagrams $(i)$ and $(i i)$ can also be seen as pullbacks in, respectively, $\mathbf{C} / D_{1}$ and $\mathbf{C} / D_{2}$. Diagram (iii) is then the image of the two pullbacks under the equivalence $\mathbf{C} / D_{1} \times \mathbf{C} / D_{2} \rightarrow \mathbf{C} /\left(D_{1}+D_{2}\right)$ of Proposition 1.3, part (i). It is a pullback since equivalences preserve limits.

\section{VAN KAMPEN SQUARES}

In this section we work in a category $\mathbf{C}$ with pullbacks. The definition of adhesive category is stated in terms of something called a van Kampen square, which can be thought of as a "well-behaved pushout", in a similar way to which coproducts can be thought of as "well-behaved" in an extensive category; essentially this means that they satisfy some of the properties of certain pushouts in the category of sets.

The name van Kampen derives from the relationship between these squares and the van Kampen theorem in topology, in its "coverings version", as presented for example in [2]. This relationship is described in detail in [18].

Definition 2.1 (van Kampen square). A van Kampen (VK) square is a pushout as in (VK1)

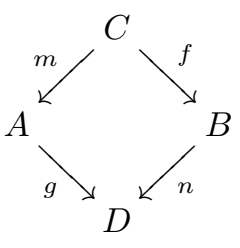

$(V K 1)$

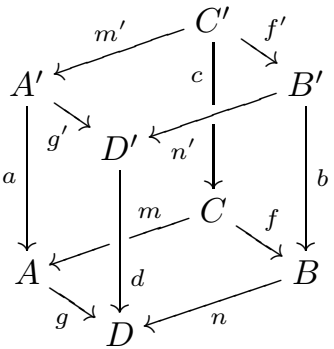

$(V K 2)$

which satisfies the following condition, for any commutative cube (VK2) of which (VK1) forms the bottom face and the back faces are pullbacks: the front faces are pullbacks if and only if the top face is a pushout. (Another way of stating the "only if" condition is that such a pushout is required to be stable under pullback.)

Another, equivalent, way of defining a VK square in a category with pullbacks is as follows. A VK square (VK1) is a pushout which satisfies the property that given a commutative diagram (i), the two squares are pullbacks if and only if there 
exists an object $C^{\prime}$ and morphisms

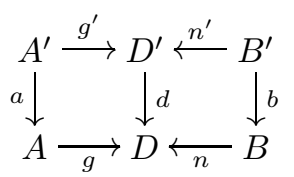

(i)

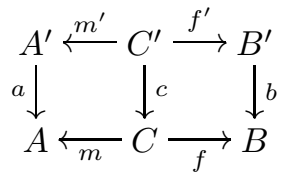

(ii)

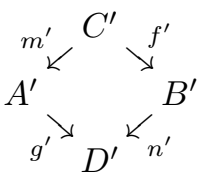

(iii)

so that the squares in (ii) are pullbacks and (iii) is a pushout.

By a pushout along a monomorphism we mean a pushout, as in diagram (VK1) above, in which $m$ is a monomorphism.

A morphism $m$ is said to be regular monomorphism when it is an equalizer

$$
A \stackrel{m}{\longrightarrow} B \stackrel{f}{\longrightarrow} C
$$

of two morphisms. (It follows easily from the universal property of equalizers that any equalizer is a monomorphism.) Then if $m$ in diagram (VK1) is a regular monomorphism, we have a pushout along a regular monomorphism, while if it is a coprojection then we have a pushout along a coprojection.

A crucial class of examples of VK squares is provided by:

Theorem 2.2. In an extensive category, pushouts along coproduct coprojections are VK squares.

Proof. If $m: C \rightarrow A$ is a coprojection, say $C \rightarrow C+E$, then the diagrams (VK1) and (VK2) have the form

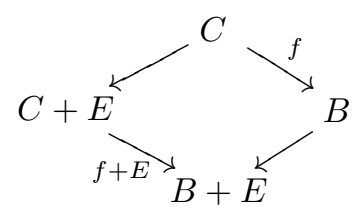

(i)

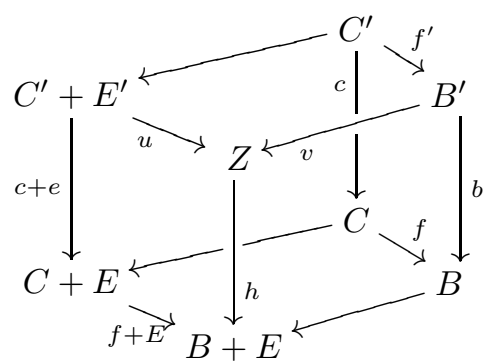

(ii)

where the unlabelled arrows are coprojections.

If the top face is a pushout then we may take $Z=B^{\prime}+E^{\prime}$, it then follows that $h=b+e$. The front right face of the cube is then a pullback, using extensivity. The front left face is a pullback using the conclusion of Lemma 1.4; it is constructed 
by "adding together" two pullbacks, namely the back right face of the cube and the pullback of the identity on $E$ and $e: E^{\prime} \rightarrow E$.

Conversely, suppose that the front faces are pullbacks. Then, as the bottom row of the following diagram

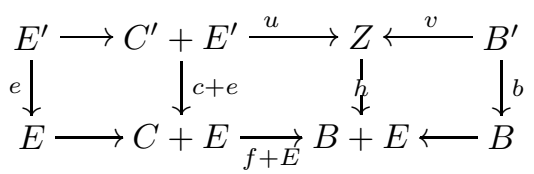

is a coproduct diagram and all the squares are pullbacks, we may deduce that the top row is a coproduct diagram, that is $Z=B^{\prime}+E^{\prime}$. Thus the top face of the cube is a pushout.

We have the following important properties of VK squares:

Lemma 2.3. In a VK square as in (VK1), if $m$ is a monomorphism then $n$ is a monomorphism and the square is also a pullback.

Proof. Suppose that the bottom face of the cube

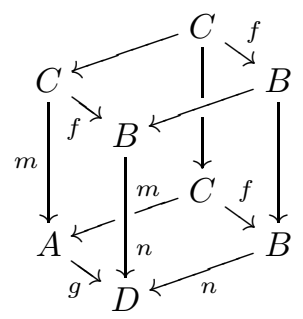

is VK. Then the top and bottom squares are pushouts, while the back squares are pullbacks if $m$ is a monomorphism. Thus the front faces will be pullbacks: the front right face being a pullback means that $n$ is a monomorphism, and the front left face being a pullback means that the original square is a pullback.

There is an alternative way of defining a VK square which involves requiring a certain functor to be an equivalence of categories. This is akin to the alternative way of presenting the main axiom of extensive categories (see Prop. 1.3).

Definition 2.4. Given a span $A \stackrel{m}{\longleftarrow} C \stackrel{f}{\longrightarrow} B$, let $\mathbf{C} / A \times_{\mathbf{C} / C} \mathbf{C} / B$ denote the category with:

- objects: commutative diagrams, as illustrated below, where both squares are pullbacks;

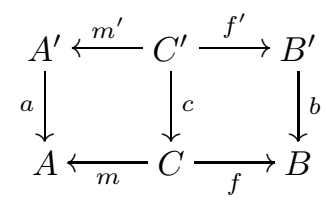


- arrows: given two such diagrams, as illustrated below, an arrow is a triple $p: A^{\prime} \rightarrow A^{\prime \prime}, q: C^{\prime} \rightarrow C^{\prime \prime}$ and $r: B^{\prime} \rightarrow B^{\prime \prime}$ so that $a^{\prime} p=a, c^{\prime} q=c$ and $b^{\prime} r=b$, while $p m^{\prime}=m^{\prime \prime} q$ and $f^{\prime \prime} q=r f^{\prime}$.

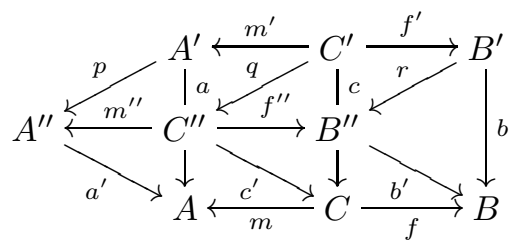

Composition and identities are obvious.

For a morphism $u: U \rightarrow V$ we shall write $u^{*}: \mathbf{C} / V \rightarrow \mathbf{C} / U$ for the functor given by pulling back along $u$. Now, suppose that we have a pushout diagram as below.

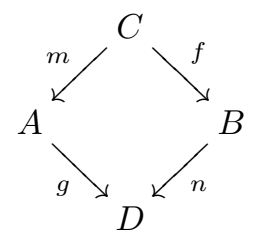

Then the functors $n^{*}$ and $g^{*}$ induce a functor

$$
\mathrm{Pb}: \mathbf{C} / D \rightarrow \mathbf{C} / A \times_{\mathbf{C} / C} \mathbf{C} / B
$$

which takes an arrow $d: D^{\prime} \rightarrow D$ and gives the object of $\mathbf{C} / A \times_{\mathbf{C} / C} \mathbf{C} / B$ obtained by taking the rear faces of the cube (VK2). One constructs the cube by taking pullbacks, first in order to construct the front faces and then the back faces. It is easy to verify that this definition defines the functor

On the other hand, if $\mathbf{C}$ has pushouts (or pushouts along monomorphisms, if we assume $m$ to be a monomorphism) we can define a functor

$$
\text { Po : } \mathbf{C} / A \times_{\mathbf{C} / C} \mathbf{C} / B \rightarrow \mathbf{C} / D
$$

as follows: starting with the back faces of diagram (VK2) we construct the pushout of $m^{\prime}$ and $f^{\prime}$ and obtain a unique arrow $d: D^{\prime} \rightarrow D$ given by the universal property of pushouts.

Proposition 2.5. $\mathrm{Pb}$ is right adjoint to $\mathrm{Po}$.

The composite $\mathrm{PoPb}$ is given by pulling back $d: D^{\prime} \rightarrow D$ and then forming a pushout; thus the counit of the adjunction is invertible if and only if, in the cube, if the vertical faces are pullbacks then the top face is a pushout; in other words, if the pushout (VK1) is stable under pullback. On the other hand, the unit of the adjunction is invertible if and only if, whenever the back faces are pullbacks, and 
the top (and bottom) faces are pushouts, then the front faces are also pullbacks. We may summarize all this as follows:

Proposition 2.6. For the pushout diagram (VK1), the following conditions are equivalent:

(i) $\mathrm{Pb}$ is an equivalence;

(ii) Po is an equivalence;

(iii) diagram (i) is a VK-square;

(iv) the pushout is stable under pullback, and the functor $\mathrm{Pb}$ is essentially surjective on objects ${ }^{2}$.

Remark 2.7. It is important to consider what underlying conditions are required of a category for $\mathrm{Pb}$ and $\mathrm{Po}$ to exist.

(i) Condition (i) of the proposition makes sense without assuming all pushouts, and could be taken as the definition of van Kampen square when not all pushouts are assumed to exist. Furthermore, if $m$ in diagram (VK1) is a monomorphism, then Po will exist provided that pushouts along monomorphisms do so, and the proposition will hold in that generality;

(ii) Condition (ii) makes sense without assuming all pullbacks, however, arbitrary pullbacks along $m$ and $g$ in the square (VK1) must exist for $\mathrm{Pb}$ to exist; recall that we make no assumptions about $g$.

We can put a preorder on monomorphisms into an object $Z$ of an arbitrary category by defining a monomorphism $a: A \rightarrow Z$ to be less than or equal to a monomorphism $b: B \rightarrow Z$ precisely when there exists an arrow $c: A \rightarrow B$ such that $b c=a$. We shall denote such a preorder by $\operatorname{Sub}(Z)$. A subobject (of $Z$ ) is an equivalence class with respect to the equivalence generated by this preorder. For example, subobjects in Set are subsets while subobjects in Graph are subgraphs. Let $g: A \rightarrow D$ be a morphism along which pullbacks of monomorphisms exist, and let $g^{*}: \operatorname{Sub}(D) \rightarrow \mathbf{S u b}(A)$ be the resulting functor. A right adjoint to $g^{*}$, if it exists, is called $\forall_{g}$. Even if $\forall_{g}$ does not exist, it may exist partially: given a subobject $C$ of $A$, we write $\forall_{g}(C)$ for a subobject of $D$ for which $D^{\prime} \leq \forall_{g}(C)$ if and only if $g^{*}\left(D^{\prime}\right) \leq C$.

Lemma 2.8. In a VK square (VK1) with $m$ a monomorphism, $n=\forall_{g}(m)$.

Proof. Let $d: D^{\prime} \rightarrow D$ be an arbitrary monomorphism, and form the cube

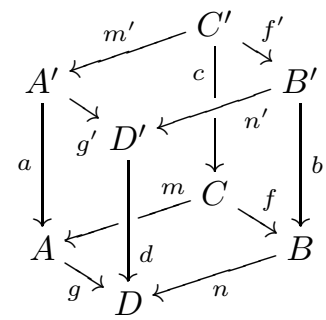

\footnotetext{
${ }^{2} \mathrm{~A}$ functor $F: \mathbf{C} \rightarrow \mathbf{D}$ is said to be essentially surjective on objects when, for every object $D \in \mathbf{D}$, there exists an object $C \in \mathbf{C}$ such that $F C \cong D$.
} 
in which the top and bottom squares are pushouts and the remaining faces are pullbacks. Observe that $g^{*}\left(d: D^{\prime} \rightarrow D\right)$ is $a: A^{\prime} \rightarrow A$. Now $D^{\prime} \leq B$ if and only if $n^{\prime}$ is invertible, while $g^{*}\left(D^{\prime}\right) \leq C$ if and only if $m^{\prime}$ is invertible. But the top face is both a pushout and a pullback, so $m^{\prime}$ is invertible if and only if $n^{\prime}$ is invertible. Thus $n: B \rightarrow D$ has the universal property of $\forall_{g}(m: C \rightarrow A)$.

\section{Adhesive CATEGORIES}

We shall now proceed to define the notion of adhesive category, and provide various examples and counterexamples.

Definition 3.1 (adhesive category). A category $\mathbf{C}$ is said to be adhesive if

(i) $\mathbf{C}$ has pushouts along monomorphisms;

(ii) C has pullbacks;

(iii) pushouts along monomorphisms are VK-squares.

\section{Remark 3.2.}

(i) By defining VK-squares as in Proposition 2.6 (i) we could leave out the assumption of pushouts along monomorphisms, but the existence of such pushouts would then be a consequence (see Rem. 2.7 (i));

(ii) Similarly, by defining VK-squares as in Proposition 2.6 (ii) we could drop the assumption of arbitrary pullbacks, but arbitrary pullbacks would then be a consequence (see Rem. 2.7 (ii)).

Just as the third axiom of extensive categories (Def. 1.2) ensures that coproducts are "well-behaved", it is the third axiom of adhesive categories which ensures that pushouts along monomorphisms are "well-behaved". This includes the fact that such pushouts are stable under pullback.

Since every monomorphism in Set is a coproduct coprojection, and Set is extensive, we immediately have:

Example 3.3. Set is adhesive.

Observe that the restriction to pushouts along monomorphisms is necessary: there are pushouts in Set which are not VK squares. Consider the underlying set of the 2-element abelian group $\mathbb{Z}_{2}$ (the argument works for any non-trivial group). In the diagram

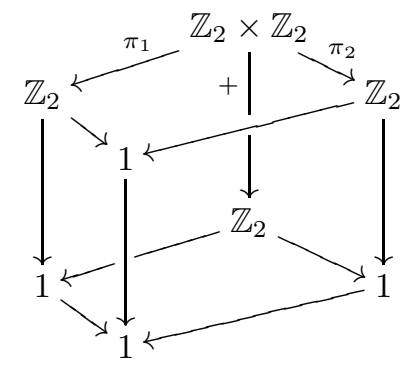


both the bottom and the top faces are easily verified to be pushouts and the rear faces are both pullbacks. However, the front two faces are not pullbacks.

Even with the restriction to pushouts along a monomorphism, many well-known extensive categories fail to be adhesive.

Example 3.4. The categories Pos, Top, Gpd and Cat are not adhesive.

Proof. Write $[n]$ for the ordered set $\{0 \leq 1 \leq \ldots n-1\}$. The pushout square

$$
\begin{aligned}
& {[1] \stackrel{0}{\rightarrow}[2]} \\
& 1 \downarrow \downarrow \\
& \text { [2] } \rightarrow[3]
\end{aligned}
$$

in Pos is not van Kampen, since it is not stable under pullback along the map $[2] \rightarrow[3]$ sending 0 to 0 and sending 1 to 2 . Thus Pos is not adhesive. The same pushout square, regarded as a pushout of categories, shows that Cat is not adhesive. For the case of $\mathbf{G p d}$, one simply replaces the poset $[n]$ by the groupoid with $n$ objects and a unique isomorphism between each pair of objects.

Finally consider the category Top of topological spaces. A finite poset induces a finite topological space on the same underlying set: the topology is determined by specifying that $y$ is in the closure of $x$ if and only if $x \leq y$. Applying this process to the previous example yields an example showing that Top is not adhesive.

Since the definition of adhesive category only uses pullbacks, pushouts, and relationships between these, we have the following constructions involving adhesive categories:

\section{Proposition 3.5.}

(i) If $\mathbf{C}$ and $\mathbf{D}$ are adhesive categories then so is $\mathbf{C} \times \mathbf{D}$.

(ii) If $\mathbf{C}$ is adhesive then so are $\mathbf{C} / C$ and $C / \mathbf{C}$ for any object $C$ of $\mathbf{C}$.

(iii) If $\mathbf{C}$ is adhesive then so is any functor category $[\mathbf{X}, \mathbf{C}]$.

Because Set is adhesive, part (iii) of the proposition implies the following.

Corollary 3.6. Any presheaf topos $[\mathbf{X}, \mathbf{S e t}]$ is adhesive.

In particular, the category Graph of directed graphs is adhesive. Indeed, if $\mathbf{C}$ is adhesive, then so is the category $\operatorname{Graph}(\mathbf{C})=[\cdot \rightrightarrows \cdot, \mathbf{C}]$ of internal graphs in $\mathbf{C}$.

Part (ii) implies that categories of typed graphs [1], coloured (or labelled) graphs [5], ranked graphs [15] and hypergraphs [11], considered in the literature on graph grammars, are adhesive.

As a consequence, all proof techniques and constructions in adhesive categories can be readily applied to any of the aforementioned categories of graphs. In fact, more generally, we have:

Proposition 3.7. Any elementary topos is adhesive.

This is somewhat harder to prove than the result for presheaf toposes; the proof can be found in [18]. 
Part (ii) of Proposition 3.5 also allows us to construct examples of adhesive categories which are not toposes.

Example 3.8. The category Set $_{*}=1 /$ Set of pointed sets (or equivalently, sets and partial functions) is adhesive, but is not extensive, and therefore, is not a topos.

Proof. In the category of pointed sets, the initial object is the one-point set 1 . Since every non-initial object has a map into 1 , the initial object is not strict, and so the category is not extensive [3], Proposition 2.8.

\section{BASIC PROPERTIES OF ADHESIVE CATEGORIES}

In this section we prove several simple lemmas which hold in any adhesive category. Lemma 4.1 describes the relationship between adhesive categories and extensive categories. Lemmas 4.2, 4.3, 4.5, 4.6 and 4.7 shed some light on pushouts in adhesive categories.

Lemma 4.1. An adhesive category is extensive if and only if it has a strict initial object.

Proof. In an extensive category the initial object is strict [3], Proposition 2.8. On the other hand, in an adhesive category with strict initial object, any arrow with domain 0 is a monomorphism. Consider the cube

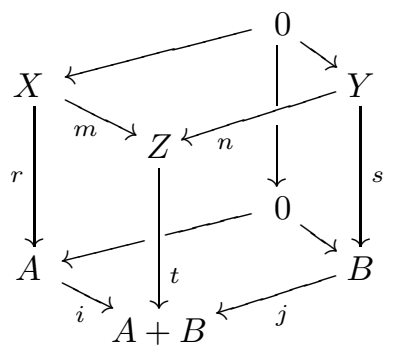

in which the bottom square is a pushout along a monomorphism, while the back squares are pullbacks since the initial object is strict. By adhesiveness, front squares are pullbacks if and only if the top squares is a pushout; but this says that the front squares are pullbacks if and only if the top row of these squares is a coproduct $(\mathrm{Z}=\mathrm{X}+\mathrm{Y})$.

As explained previously, there are adhesive categories which are not extensivefor instance Set $_{*}$ (Ex. 3.8) which doesn't have a strict initial object. Conversely, there are many extensive categories which are not adhesive; for instance Pos, Top and Cat (Ex. 3.4).

The following two lemmas are used extensively in the literature on algebraic graph rewriting. Indeed, they are usually assumed as axioms (see [9] and Sect. 8 
below) in attempts at generalizing graph rewriting. They hold in any adhesive category by Lemma 2.3 .

Lemma 4.2. Monomorphisms are stable under pushout in any adhesive category.

Lemma 4.3. In any adhesive category, pushouts along monomorphisms are also pullbacks.

The following definition introduces the notion of pushout complement [14], which is vital in algebraic approaches to graph rewriting.

Definition 4.4. Let $m: C \rightarrow A$ and $g: A \rightarrow B$ be arrows in an arbitrary category ( $m$ is not assumed to be a monomorphism).

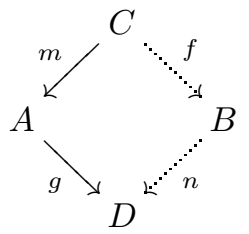

A pushout complement of the pair $(m, g)$ consists of arrows $f: C \rightarrow B$ and $n: B \rightarrow D$ for which the resulting square commutes and is a pushout, as illustrated in the diagram above. We shall sometimes refer to pushout complements of monomorphisms: this refers to pushout complements of pairs $(m, g)$ where $m$ is a monomorphism.

The following lemma is a crucial ingredient in many applications of graph rewriting. It has also been assumed as an axiom [11] in order to prove the concurrency theorem (cf. Th. 7.11). It is important mainly because it assures that once an occurrence of a left hand side of a rewrite rule is found within a structure, then the application of the rewrite rule results in a structure which is unique up to isomorphism ( $c f$. Sect. 7). In other words, rewrite rule application is functional up to isomorphism.

Lemma 4.5. Pushout complements of monomorphisms (if they exist) are unique up to isomorphism. In other words, given two pushouts along a monomorphism $m$ as illustrated below, there exists an isomorphism $\varphi: B \rightarrow B^{\prime}$ such that $n^{\prime} \varphi=n$ and $\varphi f=f^{\prime}$.
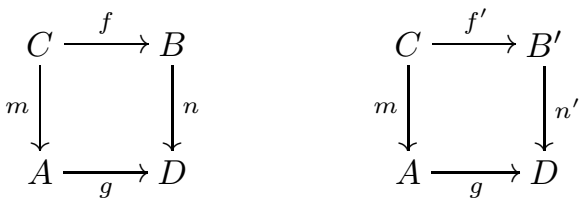

Proof. Using Lemma 2.8, both $n$ and $n^{\prime}$ have the universal property of $\forall_{g}(m)$, and so we have $n \cong n^{\prime}$ in $\operatorname{Sub}(D)$, meaning that there is an isomorphism $\varphi$ : $B \rightarrow B^{\prime}$ such that $n^{\prime} \varphi=n$. The fact that $\varphi f=f^{\prime}$ follows because $n^{\prime}$ is a monomorphism. 
The following lemma will be used in Section 8 to show that adhesive categories are high-level replacement categories:

Lemma 4.6. Consider a diagram

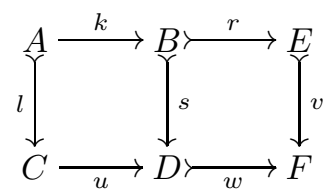

in which the marked morphisms are monomorphisms, the exterior is a pushout and the right square is a pullback. Then the left square is a pushout, and so all squares are both pullbacks and pushouts.

Proof. This amounts to stability of the exterior pushout under pullback along $w: D \rightarrow F$. We illustrate this in the diagram below, where we leave the identity morphisms unlabelled.

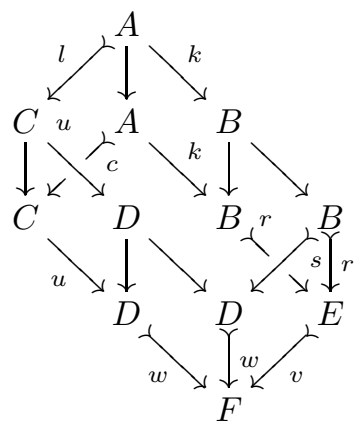

The following lemma is of a similar nature (the difference between the diagrams of Lems. 4.6 and 4.7 is that different morphisms are assumed to be mono) and was used in [24]; the proof is straightforward and similar to the proof of Lemma 4.6.

Lemma 4.7. Consider a diagram

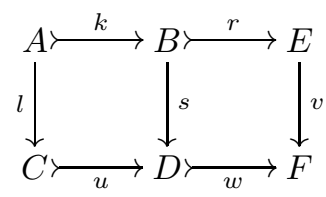

in which the marked morphisms are monomorphisms, the exterior is a pushout, the right square is a pullback, and morphisms $k, r, u$ and $w$ are monomorphisms. Then the left square is a pushout.

The following lemma shows that all monomorphisms in adhesive categories are regular. 
Lemma 4.8. Monomorphisms are regular.

Proof. Given $m: A \rightarrow B$, construct the pushout of $m$ with itself:<smiles></smiles>

Since $m$ is a monomorphism, the diagram above is also a pullback (Lem. 4.3). It is now easy to show that $m$ is the equalizer of $n_{1}$ and $n_{2}$. Indeed, suppose we're given a morphism $p: X \rightarrow B$ such that $n_{1} p=n_{2} p$. Using the pullback property, there exists a unique morphism $h: X \rightarrow A$ such that $m h=p$.

The following lemma is an easy corollary of the former, but we include a simple direct proof.

Lemma 4.9. Adhesive categories are balanced. That is, a morphism which is both a monomorphism and an epimorphism is an isomorphism.

Proof. Suppose that $f: A \rightarrow B$ is both a monomorphism and an epimorphism. Because it is an epimorphism, the diagram

$$
\begin{aligned}
& A \stackrel{f}{\longrightarrow} B \\
& \stackrel{f \downarrow}{ } \underset{B \rightarrow}{\longrightarrow} \stackrel{\downarrow}{ }{ }^{\downarrow} 1
\end{aligned}
$$

is a pushout. Since $f$ is a monomorphism, using Lemma 4.3 we can conclude that it is also a pullback, but this implies that $f$ is invertible.

\section{Algebra of subobjects}

Recall that the monomorphisms into an object $Z$ form a preorder $\operatorname{Sub}(Z)$, and that a subobject refers to an equivalence class with respect to the resulting equivalence relation. Thus the subobjects of $Z$ organise themselves in a poset. Subobject intersection (resp. union) refers to the meet (resp. join) in this poset. If a poset has both (binary) meets and joins we say that it is a lattice, a lattice is distributive when the meets distribute over the joins.

Here we shall demonstrate that, in adhesive categories, the union of two subobjects can be constructed as the pushout over their intersection. This provides further evidence of how pushouts behave in adhesive categories, as well as making more precise the intuition that the pushout operation "glues together" two structures along a common substructure. As a corollary, it follows that in an adhesive category the lattices of subobjects are distributive.

Let $\mathbf{C}$ be an adhesive category, and $Z$ a fixed object of $\mathbf{C}$. Then $\operatorname{Sub}(Z)$ has products (=intersections), given by pullback in $\mathbf{C}$. It has a top object, given 
by $Z$ itself. If $\mathbf{C}$ has a strict initial object 0 , then the unique map $0 \rightarrow Z$ is a monomorphism, and is the initial object of $\mathbf{S u b}(Z)$.

Theorem 5.1. For an object $Z$ of an adhesive category $\mathbf{C}$, the category $\mathbf{S u b}(Z)$ of subobjects of $Z$ has binary coproducts: the coproduct of two subobjects is their pushout in $\mathbf{C}$ over their intersection.

Proof. We shall show how to form binary coproducts (=unions) in $\operatorname{Sub}(Z)$. Let $a: A \rightarrow Z$ and $b: B \rightarrow Z$ be subobjects of $Z$, and form the intersection $A \cap B \rightarrow Z$, with projections $p: A \cap B \rightarrow A$ and $q: A \cap B \rightarrow B$; and now the pushout

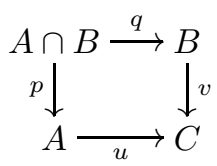

in C. Let $c: C \rightarrow Z$ be the unique map satisfying $c u=a$ and $c v=b$. We shall show that $c$ is a monomorphism, and so that $C$ is the coproduct $A \cup B$ in $\mathbf{S u b}(Z)$ of $A$ and $B$. Suppose then that $f, g: K \rightarrow C$ satisfy $c f=c g$. Form the following pullbacks
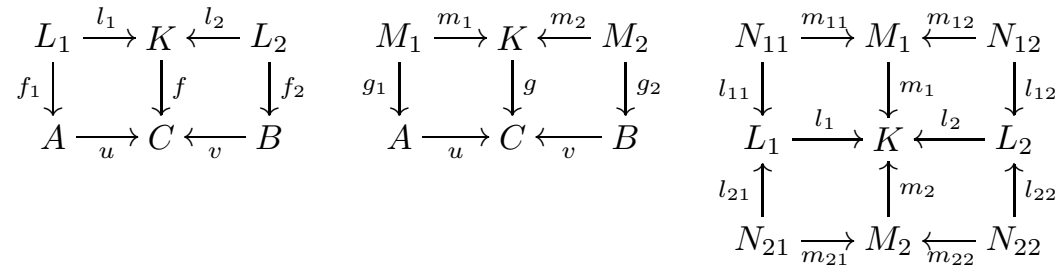

and note that each of the following pairs are the coprojections of a pushout, hence each pair is jointly epimorphic: $\left(l_{1}, l_{2}\right),\left(m_{1}, m_{2}\right),\left(m_{11}, m_{12}\right)$, and $\left(m_{21}, m_{22}\right)$. We are to show that $f=g$; to do this, it will suffice to show that $f m_{1}=g m_{1}$ and $f m_{2}=g m_{2}$; we shall prove only the former, leaving the latter to the reader. To show that $f m_{1}=g m_{1}$ it will in turn suffice to show that $f m_{1} m_{11}=g m_{1} m_{11}$ and $f m_{1} m_{12}=g m_{1} m_{12}$.

First note that $a f_{1} l_{11}=c u f_{1} l_{11}=c f l_{1} l_{11}=c g l_{1} l_{11}=c g m_{1} m_{11}=c u g_{1} m_{11}=$ $a g_{1} m_{11}$, so that $f_{1} l_{11}=g_{1} m_{11}$ since $a$ is monic; thus $f m_{1} m_{11}=f l_{1} l_{11}=u f_{1} l_{11}=$ $u g_{1} m_{11}=g m_{1} m_{11}$ as required.

On the other hand, $b f_{2} l_{12}=c v f_{2} l_{12}=c f l_{2} l_{12}=c g l_{2} l_{12}=c g m_{1} m_{12}=$ $c u g_{1} m_{12}=a g_{1} m_{12}$, so by the universal property of the pullback $A \cap B$, there is a unique map $h: N_{12} \rightarrow A \cap B$ satisfying $p h=g_{1} m_{12}$ and $q h=f_{2} l_{12}$. Now $f m_{1} m_{12}=f l_{2} l_{12}=v f_{2} l_{12}=v q h=u p h=u g_{1} m_{12}=g m_{1} m_{12}$, and so $f m_{1}=g m_{1}$ as claimed. As promised, we leave the proof that $f m_{2}=g m_{2}$ to the reader, and deduce that $f=g$, so that $c$ is monic.

Since pushouts are stable it follows that intersections distribute over unions:

Corollary 5.2. The lattice $\operatorname{Sub}(Z)$ is distributive. 
Proof. It is easy to verify that the front and back faces of the cube below are pullbacks. Because the bottom face is a pushout, we use adhesivity in order to conclude that the top face is a pushout, which in turn implies that $A \cap(B \cup C)=$ $(A \cap B) \cup(A \cap C)$.

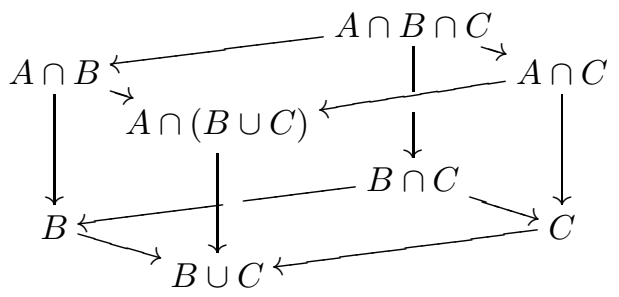

\section{Quasiadhesive Categories}

The notion of adhesivity is too strong for some relevant examples, and therefore, it is useful to study weaker notions. In this section, we introduce the class of quasiadhesive categories. Quasiadhesive categories have well-behaved pushouts along regular ${ }^{3}$ monomorphisms, that is, only such pushouts are required to be VK-squares.

Definition 6.1. A category $\mathbf{C}$ is said to be quasiadhesive if

(i) $\mathbf{C}$ has pushouts along regular monomorphisms;

(ii) C has pullbacks;

(iii) pushouts along regular monomorphisms are VK-squares.

Since regular monomorphisms are monomorphisms, the class of quasiadhesive categories includes the class of adhesive categories. In fact, we have that

Proposition 6.2. Adhesive categories are precisely the quasiadhesive categories in which every monomorphism is regular.

The conclusion of the Proposition 6.2 raises the question of whether it is useful to study the stronger property of adhesivity when quasiadhesive categories are strictly more general. We believe that the answer is positive due to the added simplicity of not having to consider regularity when it is not required.

Although quasiadhesive categories are strictly more general than adhesive categories, the categories mentioned in Example 3.4 still fail to be quasiadhesive: in each case the pushout in question is actually the pushout of two regular monomorphisms.

Just as an adhesive category is extensive iff it has a strict initial object (Lem. 4.1), we have:

Lemma 6.3. A quasiadhesive category is extensive if and only if it has a strict initial object.

\footnotetext{
${ }^{3}$ Recall that a regular monomorphism is one that is an equalizer of two morphisms.
} 


\subsection{Properties of QUASIADhesive CATEGORIES}

It will be useful to examine the properties of regular monomorphisms in quasiadhesive categories. First, recall some basic facts about regular monomorphisms which hold in any category.

Proposition 6.4. The following hold in any category $\mathbf{C}$ :

(i) if $m n$ is a regular monomorphism and $m$ is arbitrary then $n$ is a regular monomorphism;

(ii) if $\mathbf{C}$ has pullbacks then the class of regular monomorphisms is stable under pullback.

An important property of regular monomorphisms in quasiadhesive categories is that the composite of regular monomorphisms is a regular monomorphism; this is not the case in general (non-quasiadhesive) categories - for example it is not true in Cat. Another crucial property which holds in quasiadhesive categories is that regular monomorphisms are stable under pushouts.

Lemma 6.5. The following hold in any quasiadhesive category $\mathbf{C}$ :

(i) regular monomorphisms are stable under pushout;

(ii) regular monomorphisms are closed under composition.

Proof.

(i) Suppose that diagram (a) below is a pushout and that $m$ is a regular monomorphism.

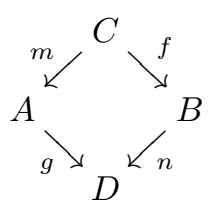

(a)

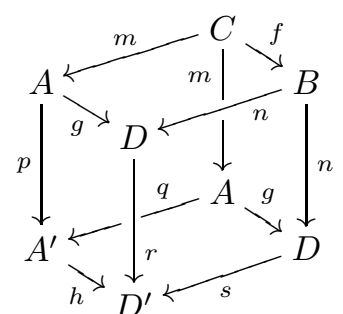

(b)

Then $m$ is the equalizer of $p$ and $q$, obtained by constructing the pushout illustrated as the back left face of diagram $(b)$. Now there exists a (unique) morphism $t: A^{\prime} \rightarrow A$ such that $t p=\operatorname{id}_{A}$ and $t q=\operatorname{id}_{A} \cdot{ }^{4}$ It follows that $p$ and $q$ are regular (for instance, $p$ is easily checked to be the equaliser of id $A_{A^{\prime}}$ and $p t$ ). Hence we are able to complete the cube in $(b)$ so that all faces are pushouts. Indeed all except possibly the front right face are

\footnotetext{
${ }^{4}$ Thus $p$ and $q$ are said are split monomorphisms; a morphism is said to be a split monomorphism precisely when it has a left inverse. It follows easily that such a morphism is mono and, moreover, regular.
} 
pushouts along regular monomorphisms, hence VK squares, hence pullbacks.

Since the bottom square is VK, it follows that the front right face is also a pullback, which implies that $n$ is the equalizer of $r$ and $s$.

(ii) Recall from the proof of Lemma 4.8 that if a pushout of a morphism with itself is also a pullback then the morphism is a regular monomorphism. Now, if $m$ and $n$ are regular monomorphisms, form the pushouts in

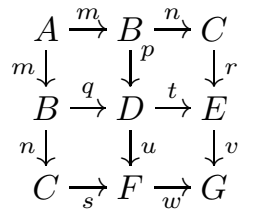

and use the fact (i) that regular monomorphisms are stable under pushout to deduce that all four squares are VK, hence pullbacks, and so that the composite square is a pullback, and so finally that $\mathrm{nm}$ is the equalizer of $v r$ and $w s$.

One also has the closure properties shown for adhesive categories. In particular:

Proposition 6.6. Let $\mathbf{C}$ and $\mathbf{D}$ be quasiadhesive categories, $\mathbf{X}$ an arbitrary category, and $C$ an object of $\mathbf{C}$. Then:

(i) $\mathbf{C} \times \mathbf{D}$ is quasiadhesive;

(ii) $\mathbf{C} / C$ is quasiadhesive.

If moreover $\mathbf{C}$ has equalizers then also

(iii) $C / \mathbf{C}$ is quasiadhesive;

(iv) the functor category $[\mathbf{X}, \mathbf{C}]$ is quasiadhesive.

Proof. We rely on the facts that pushouts and pullbacks are constructed pointwise in all of the above cases, the class of regular monomorphisms in $\mathbf{C} \times \mathbf{D}$ is the product of the regular monomorphisms of $\mathbf{C}$ and $\mathbf{D}$, the regular monomorphisms of $\mathbf{C} / C$ coincide with those of $\mathbf{C}$. We use the hypothesis that $\mathbf{C}$ has equalizers to ensure that the regular monomorphisms of $C / \mathbf{C}$ and $[\mathbf{X}, \mathbf{C}]$ are formed as in $\mathbf{C}$.

We can also say something about the subobjects in quasiadhesive categories.

Proposition 6.7. Given any two regular subobjects in the category of subobjects $\operatorname{Sub}(Z)$, their coproduct is calculated as the pushout over their intersection. In general, however, there seems to be no reason why the resulting subobject should be regular.

\subsection{EXAMPLES}

Binary relations. A simple, yet important, example of a quasiadhesive category is the category BRel of binary relations. The objects of this category are injective 
functions $m: E \rightarrow V \times V$, where $E, V$ are arbitrary sets and $V \times V$ denotes the cartesian product of $V$ with itself. Morphisms are commutative squares

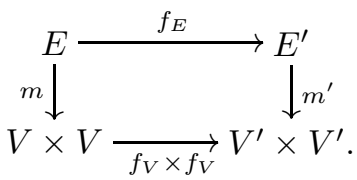

Computer scientists may be familiar with this category in another guise, that is, the category of graphs without edge identities; or in other words, graphs with at most one edge from one vertex to another. More formally, the category BRel is easily seen to be equivalent to the category $\mathbf{G r}$ where the objects are the class $\{\langle V, E\rangle \mid E \subseteq V \times V\}$ and the morphisms are ordinary graph homomorphisms.

It is routine to verify that $\mathbf{B R e l}$ is complete and cocomplete, indeed, BRel is a full reflective subcategory of Graph, the category of "ordinary" graphs and graph morphisms. Limits are calculated pointwise, and a consequence, a mono in BRel is a morphism where both $f_{E}$ and $f_{V}$ are injective. Regular monomorphisms in BRel are easily characterised as precisely the graph monomorphisms which reflect edges, or more abstractly:

Proposition 6.8. A monomorphism is regular in BRel iff the corresponding square of monos is a pullback diagram.

Notice that a simple corollary of Proposition 6.8 is that BRel is not a topos, since in toposes every monomorphism is regular. For the same reasons, it is not adhesive (Lem. 4.8). Indeed, we shall demonstrate a simple counterexample of a pushout along a mono which fails to be VK, let 1 denote the object $0 \rightarrow 1 \times 1$ (the discrete graph on one vertex) and let $1^{\prime}$ denote the object $1 \rightarrow 1 \times 1$ (the complete graph on one vertex). Then clearly, the diagram below is a pushout along a mono in BRel, but it is not a pullback.

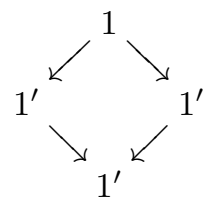

Lemma 6.9. BRel is quasiadhesive.

Proof. See Remark 6.15.

Algebraic specifications. Here we shall consider the category Spec [9] of algebraic specifications and algebraic specification morphisms. We begin by recalling the definition of Spec as given in [9]. First we recall a simple notion of multi-sorted signature for an algebraic specification.

Definition 6.10. A signature $\Sigma=\langle S, P, d o m, \operatorname{cod}\rangle$ where $S$ is a set of sorts, $P$ is a set of operator symbols, dom $: P \rightarrow S^{*}$ is a map giving each operator 
symbol a possibly empty string over $S$ and $\operatorname{cod}: P \rightarrow S$ gives each operator a sort. An operator $\sigma \in P$ with $\operatorname{dom}(\sigma)=s_{1} \ldots s_{n}$ and $\operatorname{cod}(\sigma)=s$ shall be written $\sigma: s_{1} \ldots s_{n} \rightarrow s$. A signature morphism $f: \Sigma_{0} \rightarrow \Sigma_{1}$ consists of a maps $f_{S}: S_{0} \rightarrow S_{1}$ and $f_{P}: P_{0} \rightarrow P_{1}$ so that given $\sigma: s_{1} \ldots s_{n} \rightarrow s$ we have $f_{P}(\sigma): f_{S}\left(s_{1}\right) \ldots f_{S}\left(s_{n}\right) \rightarrow f_{S}(s)$. Let $\mathbf{S i g}$ denote the category of signatures and signature morphisms.

Fixing a particular signature $\Sigma$ and a set $X=\bigcup_{s \in S} X_{s}$ of variables for each sort $s$ of $\Sigma$, one constructs the set of all terms of each sort $s$ with variables from $X$, denoted $T_{\Sigma}(X)_{s}$, in a standard way.

Definition 6.11. An equation is a pair of terms $t_{1}, t_{2} \in T_{\Sigma}(X)_{s}$, denoted $t_{1}=t_{2}$. A positive conditional equation is an expression of the form $e_{1} \wedge \cdots \wedge e_{n} \Rightarrow e^{\prime}$ where $e_{1}, \ldots, e_{n}, e^{\prime}$ are equations.

A signature morphism $f: \Sigma_{0} \rightarrow \Sigma_{1}$ induces a map $T_{\Sigma_{0}}(X)_{s} \rightarrow T_{\Sigma_{1}}(X)_{f_{S}(s)}$, also called $f$, in the obvious way. This map clearly extends to equations, so that given positive conditional equation $e$ of sort $s$ we have a positive conditional equation $f(e)$ of sort $f_{S}(s)$.

Definition 6.12. An algebraic specification $S=\langle\Sigma, E\rangle$ consists of a signature $\Sigma$ and a set $E$ of positive conditional equations over $\Sigma$. An algebraic specification morphism $f: S_{0} \rightarrow S_{1}$ consists of a signature morphism $f_{\Sigma}: \Sigma_{0} \rightarrow \Sigma_{1}$ so that for every $e \in E_{0}$ we have $f(e) \in E_{1}$. Algebraic specifications and their morphisms form the category Spec.

An algebraic specification morphism is injective if and only if the underlying signature morphism is injective - which is the same as saying the underlying functions on the sets of sorts and operators are injective. An algebraic specification morphism $f: S_{0} \rightarrow S_{1}$ is strict if, given an arbitrary positive conditional equation $e$, we have $f(e) \in E_{1}$ then $e \in E_{0}$.

Proposition 6.13. The classes of regular monomorphisms and strict injective morphisms in Spec coincide.

The reader is directed to [9] Fact 6.3.6, for details of how pushouts are constructed in Spec. Roughly, one constructs a pushout pointwise on the sorts and operators of the signature, as well as the equations. Pullbacks of arbitrary morphisms are similarly constructed "pointwise".

Lemma 6.14. Spec is quasiadhesive.

Proof. See Remark 6.15.

Remark 6.15. We have omitted the proofs of Lemmas 6.9 and 6.14. While it is possible to prove the VK property directly for pushouts along regular monos using only the descriptions of pushouts, pullbacks, and regular monos in BRel and Spec, the resulting proofs are tedious and unenlightening. Moreover, it turns out that both the examples fit into a general framework of quasiadhesive categories [18], making such proofs unnecessary. 


\section{Double-Pushout ReWriting}

Here we shall recall the basic notions of double-pushout rewriting [14,22] and show that it can be carried out within an arbitrary quasiadhesive category. In the quasiadhesive context, we work with regular monomorphisms; recall that if we happen to be in an adhesive category, every monomorphism is regular.

Definition 7.1 (production). A production $p$ is a span

$$
L \stackrel{l}{\leftarrow} K \stackrel{r}{\rightarrow} R
$$

in C. We shall say that $p$ is left-linear when $l$ is a regular monomorphism, and linear when both $l$ and $r$ are regular monomorphisms. We shall let $\mathcal{P}$ denote an arbitrary set of productions and let $p$ range over $\mathcal{P}$.

In order to develop an intuition of why a production is defined as a span, we shall restrict our attention to linear production rules. One may then consider $K$ as a substructure of both $L$ and $R$. We think of $L$ and $R$ as respectively the left-hand side and the right-hand side of the rewrite rule $p$. In order to perform the rewrite, we need to match $L$ as a substructure of a redex $C$. The structure $K$, thought of as a substructure of $L$, is exactly the part of $L$ which is to remain invariant as we apply the rule to $C$.

Thus, an application of a rewrite rule consists of three steps. First we must match $L$ as a substructure of the redex $C$; secondly, we delete all parts of the redex matched by $L$ which are not included in $K$. Thirdly, we add all of $R$ which is not contained in $K$, thereby producing a new structure $D$. The deletion and addition of structure is handled, respectively, by finding a pushout complement and constructing a pushout.

Definition 7.2 (gluing conditions). Given a production $p$ as in (1), a match in $C$ is a morphism $f: L \rightarrow C$. A match $f$ satisfies the gluing conditions with respect to $p$ precisely when there exists an object $E$ and morphisms $g: K \rightarrow E$ and $v: E \rightarrow C$ such that

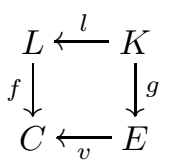

is a pushout diagram. (In other words, there exists a pushout complement of $(l, f)$ in the sense of Def. 4.4.)

Definition 7.3 (derivation). Given an object $C \in \mathbf{C}$ and a set of productions $\mathcal{P}$, we write $C \longrightarrow \longrightarrow_{p, f} D$ for a production $p \in \mathcal{P}$ and a morphism $f: L \rightarrow C$ if (a) $f$ satisfies the gluing conditions with respect to $l$, and (b) there is a diagram

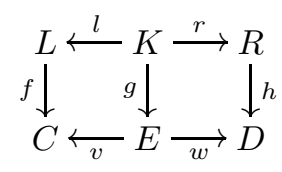

in which both squares are pushouts. 
The object $E$ in the above diagram can be thought of as a temporary state in the middle of the rewrite process. Returning briefly to our informal description, it is the structure obtained from $C$ by deleting all the parts of $L$ not contained in $K$. Recall from Lemma 4.5 that if $l$ is a regular monomorphism (that is, if $p$ is leftlinear) then $E$ is unique up to isomorphism. Indeed, if $p$ is a left-linear production, $C \longrightarrow{ }_{p, f} D$ and $C \longrightarrow{ }_{p, f} D^{\prime}$ then we must have $D \cong D^{\prime}$. This is a consequence of Lemma 4.5 and the fact that pushouts are unique up to isomorphism.

Definition 7.4 (adhesive and quasiadhesive grammars). A quasiadhesive grammar $\mathbf{G}$ is a pair $\langle\mathbf{C}, \mathbf{P}\rangle$ where $\mathbf{C}$ is a quasiadhesive category and $\mathbf{P}$ is a set of linear productions. If $\mathbf{C}$ is in fact adhesive, we call $\mathbf{G}$ an adhesive grammar.

Assuming that all the productions are linear allows us to derive a rich rewriting theory on quasiadhesive categories. Henceforth we assume that we are working over an arbitrary quasiadhesive grammar $\mathbf{G}$.

\subsection{Local Church-Rosser theorem}

As we shall explain in Section 8, adhesive and quasiadhesive categories naturally fall into the framework of high-level replacement categories. In particular, we get the local Church-Rosser theorem $[9,16]$ in the setting of quasiadhesive or adhesive grammars.

Before presenting this theorem we recall briefly the notions of parallelindependent derivation and sequential-independent derivation. The reader may wish to consult [5] for a more complete presentation.

Definition 7.5. A parallel-independent derivation is a pair of derivations

$$
C \longrightarrow p_{p_{1}, f_{1}} D_{1} \quad \text { and } \quad C \longrightarrow p_{p_{2}, f_{2}} D_{2}
$$

as illustrated in diagram (2) which satisfy an additional requirement, namely the existence of morphisms $r: L_{1} \rightarrow E_{2}$ and $s: L_{2} \rightarrow E_{1}$ which render the diagram commutative, in the sense that $v_{2} r=f_{1}$ and $v_{1} s=f_{2}$.

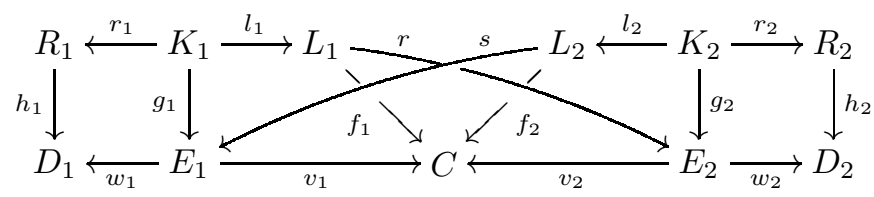

Definition 7.6. Similarly, a sequential-independent derivation, illustrated in diagram (3), is a derivation

$$
C \longrightarrow p_{p_{1}, f_{1}} D_{1} \longrightarrow p_{p_{2}, f_{2}^{\prime}} D
$$


where there additionally exist arrows $r^{\prime}: R_{1} \rightarrow E_{3}$ and $s^{\prime}: L_{2} \rightarrow E_{1}$ such that $w_{1} s^{\prime}=f_{2}^{\prime}$ and $v_{3} r^{\prime}=h_{1}$.

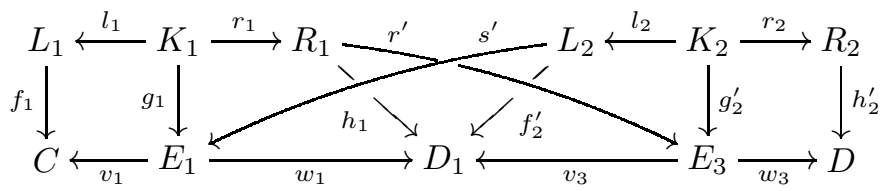

If the underlying category has coproducts and given a pair of productions

$$
p_{1}=L_{1} \stackrel{l_{1}}{\longleftarrow} K_{1} \stackrel{r_{1}}{\longrightarrow} R_{1} \quad \text { and } \quad p_{2}=L_{2} \stackrel{l_{2}}{\longleftarrow} K_{2} \stackrel{r_{2}}{\longrightarrow} R_{2},
$$

we may construct a production

$$
p_{1}+p_{2}=L_{1}+L_{2} \stackrel{l_{1}+l_{2}}{\longleftarrow} K_{1}+K_{2} \stackrel{r_{1}+r_{2}}{\longrightarrow} R_{1}+R_{2} .
$$

Note that if $l_{1}$ and $l_{2}$ are regular monomorphisms, $l_{1}+l_{2}$ need not even be a monomorphism, and similarly for $r_{1}$ and $r_{2}$. If this is required one could further suppose $\mathbf{C}$ to be extensive, in which case the coproduct of two regular monomorphisms would indeed be a regular monomorphism.

The statement of the theorem below differs from those previously published in the literature in that we do not need coproducts to establish the equivalence of the first 3 items.

Theorem 7.7 (local Church-Rosser). The following are equivalent:

(1) $C \longrightarrow p_{p_{1}, f_{1}} D_{1}$ and $C \longrightarrow p_{p_{2}, f_{2}} D_{2}$ are parallel-independent derivations;

(2) $C \longrightarrow p_{1}, f_{1} D_{1}$ and $D_{1} \longrightarrow p_{2}, f_{2}^{\prime} D$ are sequential-independent derivations;

(3) $C \longrightarrow p_{p_{2}, f_{2}} D_{2}$ and $D_{2} \longrightarrow p_{p_{1}, f_{1}^{\prime}} D$ are sequential-independent derivations.

If moreover $\mathbf{C}$ has coproducts then we may add the so-called parallelism theorem, which states that item (4), below, is equivalent to the above three items.

(4) $C \longrightarrow p_{1}+p_{2},\left[f_{1}, f_{2}\right] D$ is a derivation.

Proof. (1) $\Rightarrow(2)$ : Let $C \longrightarrow p_{p_{1}, f_{1}} D_{1}$ and $C \longrightarrow p_{p_{2}, f_{2}} D_{2}$ be parallel-independent (see Def. 7.5 and associated diagram) derivations. Form the pullback (i) below.

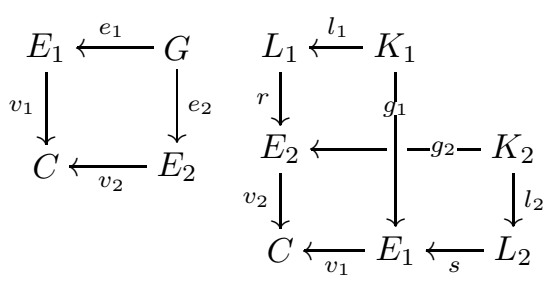

(i)

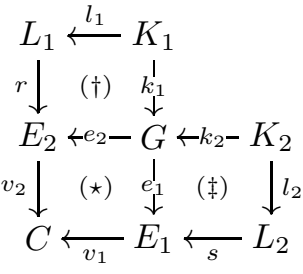

(iii)

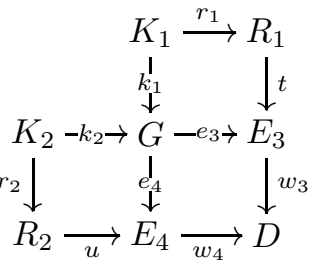

(iv) 
The two regions in (ii) are pushouts [ $f$. diagram (3)]. Combining the two diagrams gives (iii), with $k_{1}$ and $k_{2}$ obtained by the universal property of (i) and satisfying $e_{2} k_{1}=g_{1}$ and $e_{1} k_{2}=g_{2}$. Regions $(\dagger),(\ddagger)$, and $(\star)$ are pushouts by Lemma 4.6, and one now goes on to construct (iv) by taking successive pushouts.

The sequential-independent (see Def. 7.6 and associated diagram) derivation $C \longrightarrow p_{1}, f_{1} D_{1} \longrightarrow p_{p_{2}, f_{2}^{\prime}} D$ may now be constructed with the pushout squares below.
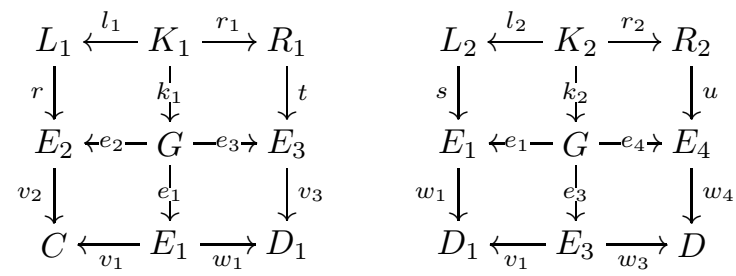

$(2) \Rightarrow(1):$ Suppose that $C \longrightarrow p_{p_{1}, f_{1}} D_{1}$ and $D_{1} \longrightarrow_{p_{2}, f_{2}^{\prime}} D$ are sequentialindependent derivations. Form the pullback $(v)$ below.<smiles></smiles>

$(v)$

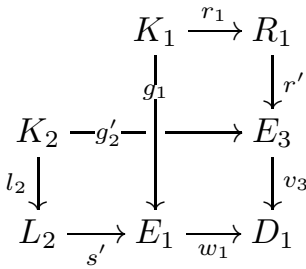

$(v i)$

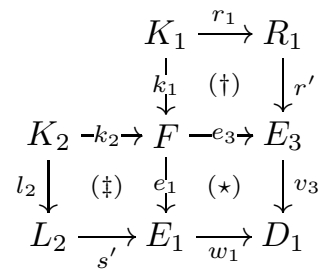

(vii)

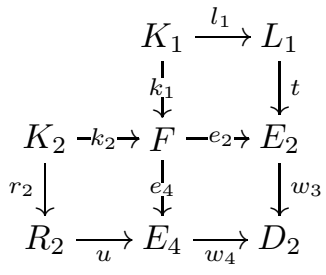

(viii)

The two regions in (vi) are pushouts $[c f$. diagram (3)]. Combining the two diagrams gives (vii), with $k_{1}$ and $k_{2}$ obtained by the universal property of $(i)$ and satisfying $e_{3} k_{2}=g_{2}^{\prime}$ and $e_{1} k_{1}=g_{1}$. Regions $(\dagger),(\ddagger)$, and $(\star)$ are pushouts by Lemma 4.6, and one now goes on to construct (viii) by taking successive pushouts.

The parallel independent derivations $C \longrightarrow{ }_{p_{1}, f_{1}} D_{1}$ and $C \longrightarrow p_{p_{2}, f_{2}} D_{2}$ may now be constructed with the pushout squares below.

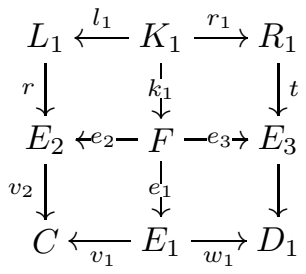

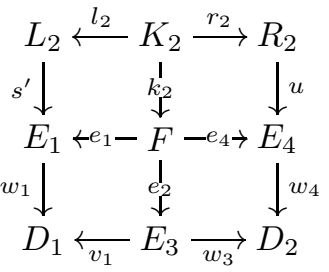

In fact, the proof that $(1) \Rightarrow(2)$ remains valid more generally in the context of left-linear productions, but the proof of the converse requires linearity.

The proof of $(1) \Leftrightarrow(3)$ is similar.

To prove $(1) \Leftrightarrow(4)$, we first note that, in a quasiadhesive category with coproducts, the objects $C$ and $D$ obtained in (iii) and (iv) can be obtained as the pushouts 
in $(i x)$ and $(x)$ below.

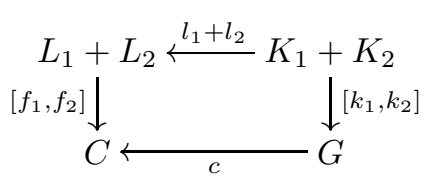

$(i x)$

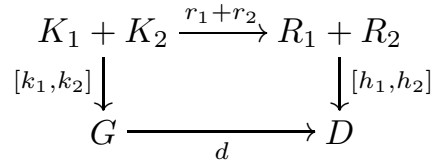

$(x)$

Starting with parallel-independent derivations $C \longrightarrow p_{p_{1}, f_{1}} D_{1}$ and $C \longrightarrow p_{p_{2}}, f_{2}$ $D_{2}$, we construct diagrams (iii) and (iv) from which, using the translation outlined above, we get diagrams $(i x)$ and $(x)$, which in turn amount to a derivation $C$ $\triangleright_{p_{1}+p_{2},\left[f_{1}, f_{2}\right]} D$. Similarly, starting with a derivation $C \longrightarrow p_{p_{1}+p_{2},\left[f_{1}, f_{2}\right]} D$, we obtain diagram $(i x)$, from which we obtain diagram (iii). This gives us enough data to conclude that $C \longrightarrow p_{p_{1}, f_{1}} D_{1}$ and $C \longrightarrow{ }_{p_{2}, f_{2}} D_{2}$ are parallel-independent.

\subsection{Concurrency theOrem}

The original concurrency theorems were proved for graph grammars [7] and later generalized to high-level replacement categories ( $c f$. Sect. 8) in [11] which satisfy additional axiom sets, there called HLR2 and HLR2*. Roughly, the concurrency theorem states that given two derivations in a sequence, together with information about how they are related, one may construct a single derivation which internalizes the two original derivations and performs them "concurrently". Moreover, one may reverse this process and deconstruct a concurrent derivation into two related sequential derivations. Here we state and prove the concurrency theorem for quasiadhesive or adhesive grammars without the need for extra axioms.

We shall first need to recall the notions of dependency relation, dependent derivation and concurrent production.

Definition 7.8 (dependency relation). Suppose that $p_{1}$ and $p_{2}$ are linear productions. A dependency relation for $\left\langle p_{1}, p_{2}\right\rangle$ is an object $X$ together with arrows $s: X \rightarrow R_{1}$ and $t: X \rightarrow L_{2}$ for which $r_{1}, s, t$, and $l_{2}$ can be incorporated into a diagram

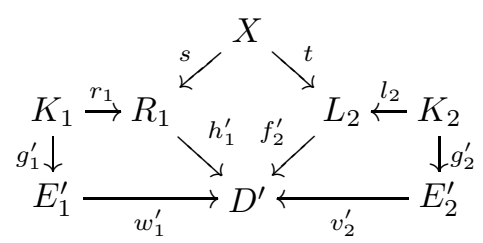

in which all three regions are pushouts. 
Definition 7.9 (dependent derivation). Consider a derivation $C \longrightarrow p_{p_{1}, f_{1}} D_{1}-$ $\triangleright_{p_{2}, f_{2}} D$ as illustrated in (i) below

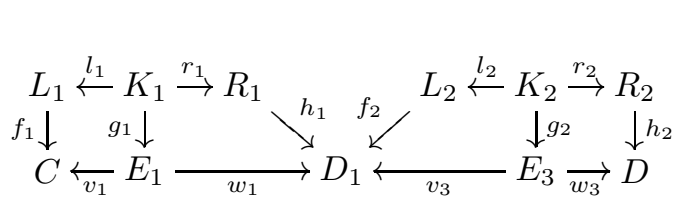

(i)

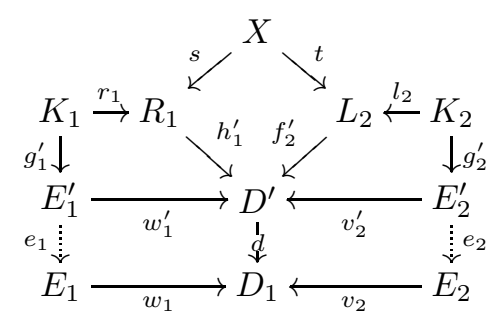

(ii)

and a dependency relation $X$ for $\left\langle p_{1}, p_{2}\right\rangle$. The derivation is said to be $X$-dependent if $h_{1} s=f_{2} t$ and there exist morphisms $e_{1}: E_{1}^{\prime} \rightarrow E_{1}$ and $e_{2}: E_{2}^{\prime} \rightarrow E_{2}$ satisfying $e_{1} g_{1}^{\prime}=g_{1}$ and $e_{2} g_{2}^{\prime}=g_{2}$, and if moreover the unique map $d: D^{\prime} \rightarrow D_{1}$ satisfying $d h_{1}^{\prime}=h_{1}$ and $d f_{2}^{\prime}=f_{2}$ also satisfies $d w_{1}^{\prime}=w_{1} e_{1}$ and $d v_{2}^{\prime}=v_{2} e_{2}$ (see (ii)).

Definition 7.10 (concurrent production). Given a dependency relation $X$ for $\left\langle p_{1}, p_{2}\right\rangle$, the $X$-concurrent production $p_{1} ; x p_{2}$ is the span

$$
C^{\prime} \stackrel{v_{1}^{\prime} u^{\prime}}{\longleftarrow} P^{\prime} \stackrel{w_{2}^{\prime} v^{\prime}}{\longrightarrow} D^{\prime}
$$

obtained by taking the bottom row of the following extension of diagram (4)

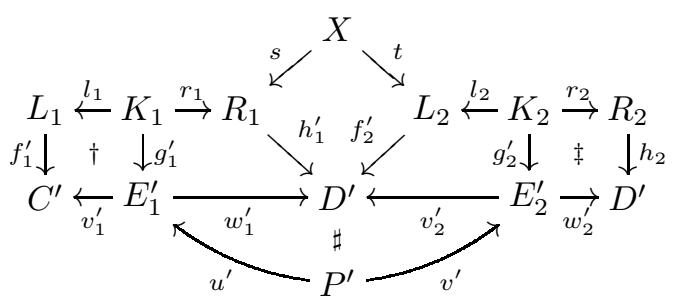

in which $\dagger$ and $\ddagger$ are pushouts and $\sharp$ is a pullback.

An $X$-concurrent derivation $C \longrightarrow p_{p_{1} ; x} p_{2}, f$ is a derivation where $p_{1} ; X p_{2}$ is an $X$-concurrent production.

Theorem 7.11 (concurrency theorem).

(1) Given an $X$-dependent derivation $C \longrightarrow{ }_{p_{1}, f_{1}} D_{1} \longrightarrow{ }_{p_{2}, f_{2}} D$ there exists an $X$-concurrent derivation $C \longrightarrow p_{1} ; p_{2} D$.

(2) Given an $X$-concurrent derivation $C \longrightarrow p_{p_{1} ; x} p_{2} D$, there exists an $X$ dependent derivation $C \longrightarrow p_{p_{1}, f_{1}} D_{1} \longrightarrow p_{p_{2}, f_{2}} D$. 
Proof. (1). Suppose that we have an $X$ dependent-derivation, as illustrated in the solid part of the left diagram below
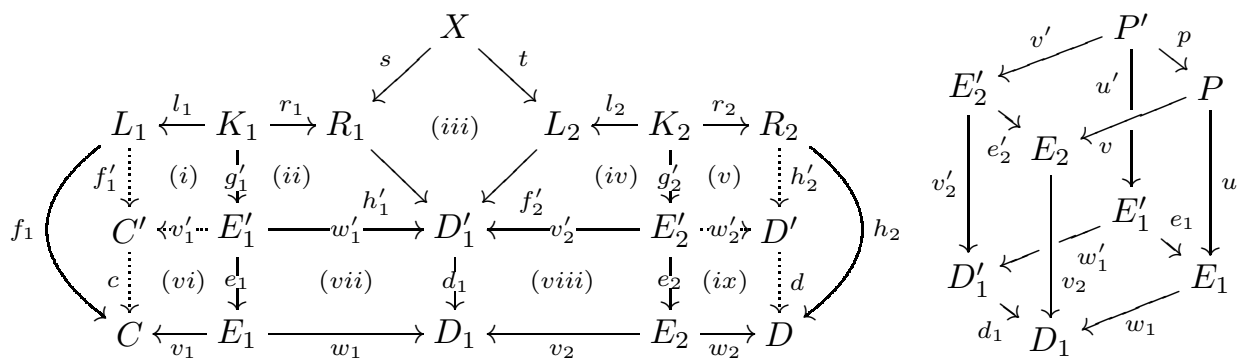

in which (iii), $(i)+(v i),(i i)+(v i i),($ iv $)+(v i i i),(v)+(i x),(i i)$, and (iv) are pushouts, so that also (vii) and (viii) are pushouts. Fill in the dotted parts of the diagram to obtain further pushouts (i), (v), (vi), and (ix).

By Lemma 4.2 both $w_{1}^{\prime}$ and $v_{2}^{\prime}$ are monomorphisms, and now by Lemma 4.3 both (vii) and (viii) are pullbacks. Consider the cube, above right, in which bottom and front left faces are the pullbacks (vii) and (viii), and the remaining faces are constructed so as to be pullbacks. Since the bottom face is also a pushout, so is the top face. Similarly, since the front left face is a pushout, so is the back right face. Combining the top and back right faces of the cube with regions (vi) and

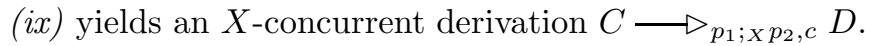

(2). Suppose that we have an $X$-concurrent derivation $C \longrightarrow{ }_{p_{1} ; x p_{2}, f} D$, as illustrated by the solid part of the diagram below left.

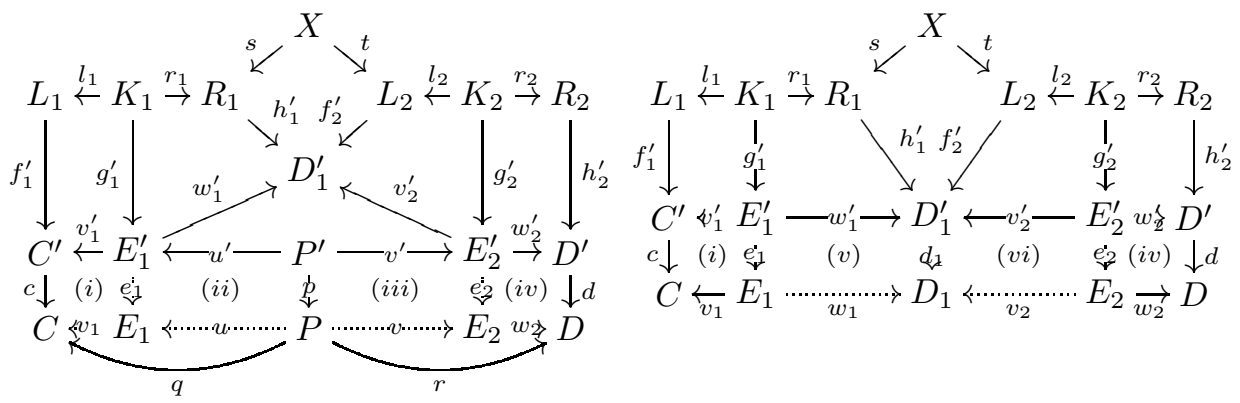

We construct pushouts (ii) and (iii) and obtain $v_{1}$ and $w_{2}$ using the universal properties. It now follows that (i) and (iv) are also pushouts. Now construct the pushout $(v)$; since $(i i)+(v)$ and (iii) are pushouts, there is a unique map $v_{2}: E_{2} \rightarrow D_{1}$ so that (vi) is a pushout and $(i i i)+(v i)$ equals $(i i)+(v)$. The diagram on the right now provides the required $X$-dependent derivation $C \longrightarrow p_{p_{1}, c f_{1}^{\prime}} D_{1} \longrightarrow p_{p_{2}, d_{1} f_{2}^{\prime}} D$. 


\section{Relationship With High-LEVEL REPlaCEMENT CATEGORIES}

High-level replacement categories [9-11] or HLR-categories encompass several attempts to isolate general categorical axioms which lead to categories in which one can define double-pushout graph rewriting and prove useful theorems such as the local Church-Rosser theorem and the concurrency theorem.

Definition 8.1 (HLR-categories). A category $\mathbf{S}$ with a class of morphisms $\mathcal{M}$ is an HLR-category if it satisfies the following axioms:

(1) pairs $C \leftarrow A \rightarrow B$ with at least one of the arrows in $\mathcal{M}$ have a pushout;

(2) pairs $B \rightarrow D \leftarrow C$ with both morphisms in $\mathcal{M}$ have pullbacks;

(3) $\mathcal{M}$ is stable under pushout;

(4) $\mathcal{M}$ is stable under pullback;

(5) finite coproducts exist;

(6) if $m$ and $n$ are in $\mathcal{M}$ then $m+n$ is in $\mathcal{M}$;

(7) pushouts of morphisms in $\mathcal{M}$ are pullbacks;

(8) pushout-pullback decomposition holds: that is, given a diagram

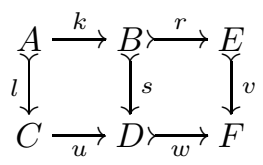

if the marked morphisms are in $\mathcal{M}$, the whole rectangle is a pushout and the right square is a pullback, then the left square is a pushout.

Lemma 8.2. An adhesive category with a strict initial object is an HLR-category, with $\mathcal{M}$ the class of monomorphisms.

Proof. This follows immediately from Lemmas 4.1, 4.2, 4.3, and 4.6.

Similarly, quasiadhesive categories can be seen as HLR-categories:

Lemma 8.3. A quasiadhesive category with a strict initial object is an HLRcategory, with $\mathcal{M}$ the class of regular monomorphisms.

Proof. Immediate by Lemmas 6.3 and 6.5 , in addition to the "quasi" versions of Lemmas 4.3 and 4.6 .

In both Lemmas 8.2 and 8.3, the strict initial object is needed only to guarantee extensivity, which in turn is needed only in order for axioms (5) and (6) to hold.

The axioms listed above are enough to prove the local Church-Rosser theorem (cf. Th. 7.7), but not the concurrency theorem ( $c f$. Th. 7.11). To prove the latter, extra axioms had to be introduced in [11], such as the conclusion of the following lemma. Interestingly, it is almost the dual of the main axiom of adhesive categories.

Lemma 8.4 (cube-pushout-pullback-lemma [11]). Given a cube in which all arrows in the top and bottom faces are monomorphisms, if the top face is a pullback 
and the front faces are pushouts, then the bottom face is a pullback if and only if the back faces are pushouts.

Proof. Since the front faces are pushouts along monomorphisms, they are also pullbacks.

If the bottom face is a pullback, then the back faces are pushouts by stability of the pushouts on the front faces. Suppose conversely that the back faces are pushouts; since they are pushouts along monomorphisms, they are also pullbacks. One now simply "rotates the cube": since the front right and back left faces are pushouts, and the top and back right faces are pullbacks, it follows by adhesiveness that the bottom square is a pullback.

Clearly, one can prove a version of Lemma 8.4 for quasiadhesive categories by replacing monomorphisms with regular monomorphisms. An HLR-category which has the conclusion of Lemma 8.4 as an additional axiom is sometimes referred to as an HLR2-category [11]. It is immediate, therefore, that any adhesive category with a strict initial object is an HLR2-category $(\mathcal{M}=$ monomorphisms $)$ and similarly, that any quasiadhesive category with a strict initial object is an HLR2-category $(\mathcal{M}=$ regularmonomorphisms $)$.

The strongest axiom system for general rewriting is enjoyed by the so-called HLR2*-categories [11]. These are HLR2-categories which, additionally, have the conclusion of Lemma 4.5 as an axiom, that is, pushout complements of monomorphisms are, if they exist, unique up to isomorphism. Finally, they satisfy an axiom known as the twisted-triple-pushout condition. We believe that this axiom does not hold in arbitrary adhesive or quasiadhesive categories, although it does hold, for instance, in any topos. Indeed, it is possible to extend the definition of adhesive and quasiadhesive categories in a natural way so that the twisted-triple-pushoutcondition holds. We treat the adhesive version of the lemma below, the reader will obtain the quasiadhesive version by replacing every occurrence of "adhesive" with "quasiadhesive" and "monomorphism" with "regular monomorphism".

Lemma 8.5. If $\mathbf{C}$ is adhesive and, additionally, all pushouts are stable under pullback then $\mathbf{C}$ satisfies the twisted-triple-pushout condition: that is, given a diagram

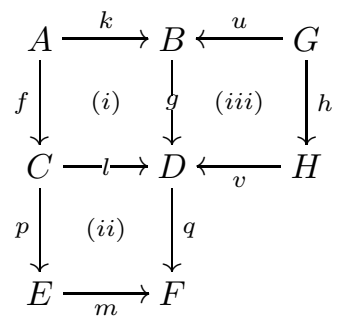

with $k, l$ and $m$ monomorphisms, (i) is a pushout when (i) +(ii) a pushout, (ii) a pullback, (iii) a pushout and $u, h$ forming a pullback of $q g$ and $q v$. 
Proof. First note that, using Lemma 4.3, $(i)+(i i)$ is a pullback. Using the pullback version of Lemma 1.1 it follows that (i) is a pullback. It is easy to verify that (iii) is a pullback.

Consider diagram (iv) below. By assumption, the front right face is a pullback, while the front left face is $(i)+(i i)$, a pushout.

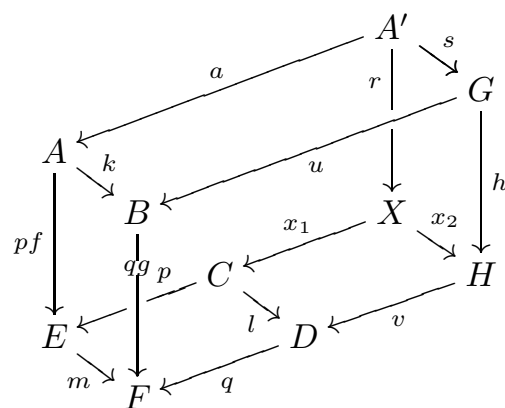

$(i v)$

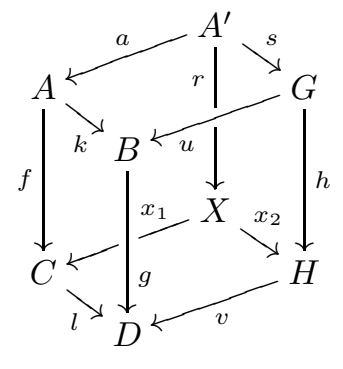

$(v)$

Furthermore, the leftmost half of the bottom face is (ii) which is, by assumption, a pullback. We obtain $X$ and morphisms $x_{1}: X \rightarrow C$ and $x_{2}: X \rightarrow H$ by taking the pullback of $l: C \rightarrow D$ and $v: H \rightarrow D$. We can now complete the diagram with an object $A^{\prime}$, and morphisms $a: A^{\prime} \rightarrow A, r: A^{\prime} \rightarrow X$ and $s: A^{\prime} \rightarrow G$ so that the back left face and the top face are pullbacks. Using adhesivity, the back right face is a pushout.

We obtain diagram $(v)$ by "cutting off" the bottom left corner of diagram (iv). The front left face is ( $i$ ), the front right face (iii), which is, by assumption, both a pushout and a pullback. The bottom and back right faces are pullbacks, since they were constructed as such in diagram (iv). To see that the back left face is commutative, note that $l f a=g k a=g u s=v h s=v x_{2} r=l x_{1} r$ and use the fact that $l$ is a monomorphism. It is also a pullback, using the pullback version of Lemma 1.1. Using the fact that arbitrary pushouts are stable under pullback, we can conclude that the back left face is a pushout. Summarizing, we have assumed that the front right face is a pushout and deduced that both the back left and right faces are pushouts. It follows from the pushouts version of Lemma 1.1 that the front left face, (i), is a pushout.

Finally, the following lemma has been used by Ehrig and König in their work on rewriting via borrowed contexts [12]. Here we prove that it holds in any adhesive category. A quasiadhesive version of the lemma can be obtained in the obvious way. 
Lemma 8.6. Given diagram (i) below with the marked morphisms monomorphisms,

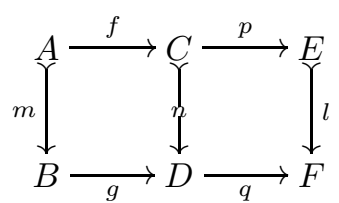

suppose that the left square is a pushout and the exterior is a pullback. Then the right square is a pullback.

Proof. Suppose we have an object $X$ and morphisms $\alpha: X \rightarrow D$ and $\beta: X \rightarrow E$ such that $q \alpha=l \beta$. We will show that there exists $k: X \rightarrow C$ such that $n k=\alpha$; then $p k=\beta$ follows since $l$ is a monomorphism, while uniqueness follows since $n$ is a monomorphism. We begin by constructing the following cube by taking pullbacks.

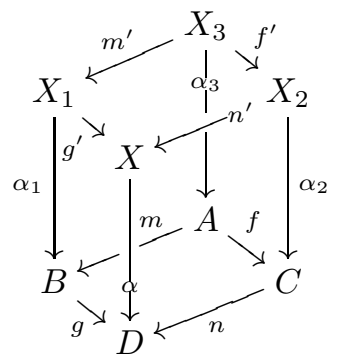

Now $q g \alpha_{1}=q \alpha g^{\prime}=l \beta g^{\prime}$; but the diagram in the statement of the lemma is a pullback, so there is a unique morphism $h: X_{1} \rightarrow A$ such that $m h=\alpha_{1}$ and $p f h=\beta g^{\prime}$.

Note that $m \alpha_{3}=\alpha_{1} m^{\prime}=m h m^{\prime}$, and using the fact that $m$ is a monomorphism, $\alpha_{3}=h m^{\prime}\left({ }^{*}\right)$. We shall use the fact that the top face of the cube is a pushout to derive the existence of the required morphism. Indeed, we have $\alpha_{2} f^{\prime}=f \alpha_{3}=$ $f h m^{\prime}$ where we used $\left(^{*}\right)$ to derive the last equality. Thus we get a unique $k: X \rightarrow$ $C$ such that $k g^{\prime}=f h$ and $k n^{\prime}=\alpha_{2}$.

It remains to show that $n k=\alpha$. Indeed, we have $n k g^{\prime}=n f h=g m h=g \alpha_{1}=$ $\alpha g^{\prime}$ and $n k n^{\prime}=n \alpha_{2}=\alpha n^{\prime}$. Using the fact that $g^{\prime}$ and $n^{\prime}$ are jointly epimorphic yields the required equality.

\section{CONClusions AND FUture WORK}

We introduced the notions of van Kampen (VK) square and adhesive category, as well as the more general quasiadhesive categories. VK squares are "well-behaved pushouts", and a category is adhesive when pushouts along monomorphisms are VK. Adhesive and quasiadhesive categories are closely related to extensive categories.

Double-pushout (DPO) rewriting can be defined in an arbitrary adhesive category. We introduced adhesive grammars, which are adhesive categories with a set 
of linear productions. Adhesive grammars have sufficient structure for the development of a rich rewriting theory. In particular, we proved the local Church-Rosser and the so-called concurrency theorem within the setting of adhesive grammars. We have also shown that adhesive categories satisfy many of the axioms $[9,11]$ which were proposed in order to prove these theorems. Thus, we have arrived at a class of categories which supports such a theory of DPO rewriting, however, we believe that adhesive categories are mathematically elegant and less ad hoc than previous proposals.

In order to back this claim and to further develop the theory of adhesive categories, we have demonstrated a number of useful properties. For instance, subobject union is formed as a pushout over the intersection, and subobject intersection distributes over subobject union. We have provided some closure properties which allow the construction of new adhesive categories from old. Any elementary topos is adhesive, but there are examples of adhesive categories which are not toposes. Adhesive categories include many well-known notions of graph structures used in computer science and are instances of HLR2-categories [11].

We believe that adhesive categories will be useful in the development of specific graphical models of computation and the development of semantic techniques for reasoning about such models. The rewriting theory needs to be developed further, with, for example, the construction of canonical dependency relations from derivations [11].

Another direction for future work is to examine whether adhesive categories have enough structure so that groupoidal relative pushouts [23] can be constructed in cospan bicategories over adhesive categories. Such cospan bicategories provide a way of understanding graphs in a modular fashion and will provide a general class of models which should include bigraphs [20] as examples. Some progress in this direction has already been achieved [24].

Acknowledgements. The second author would like to thank Vladimiro Sassone for many discussions in the early stages of this project. Thanks also go to the anonymous referees for reading early drafts and providing many valuable comments and suggestions.

\section{REFERENCES}

[1] P. Baldan, A. Corradini, H. Ehrig, M. Löwe, U. Montanari and F. Rossi, Concurrent semantics of algebraic graph transformations, in Handbook of Graph Grammars and Computing by Graph Transformation, edited by H. Ehrig, H.-J. Kreowski, U. Montanari and G. Rozenberg, World Scientific 3 (1999) 107-187.

[2] R. Brown and G. Janelidze, Van Kampen theorems for categories of covering morphisms in lextensive categories. J. Pure Appl. Algebra 119 (1997) 255-263.

[3] A. Carboni, S. Lack and R.F.C. Walters, Introduction to extensive and distributive categories. J. Pure Appl. Algebra 84 (1993) 145-158.

[4] L. Cardelli, Bitonal membrane systems. Draft (2003). 
[5] A. Corradini, H. Ehrig, R. Heckel, M. Lowe, U. Montanari and F. Rossi, Algebraic approaches to graph transformation part i: Basic concepts and double pushout approach, in Handbook of Graph Grammars and Computing by Graph Transformation, edited by G. Rozenberg, World Scientific 1 (1997) 162-245.

[6] V. Danos and C. Laneve, Graphs for core molecular biology, in International Workshop on Computational Methods in Systems Biology, CMSB '03 (2003).

[7] H. Ehrig, Introduction to the algebraic theory of graph grammars, in 1st Int. Workshop on Graph Grammars, Springer Verlag. Lect. Notes Comput. Sci. 73 (1979) 1-69.

[8] H. Ehrig, G. Engels, H.-J. Kreowski and G. Rozenberg, editors, Handbook of Graph Grammars and Computing by Graph Transformation, Volume 2: Applications, Languages and Tools. World Scientific (1999).

[9] H. Ehrig, M. Gajewsky and F. Parisi-Presicce, High-level replacement systems with applications to algebraic specificaitons and Petri Nets, in Handbook of Graph Grammars and Computing by Graph Transformation, edited by H. Ehrig, H.-J. Kreowsky, U. Montanari and G. Rozenberg, World Scientific 3 (1999) 341-400.

[10] H. Ehrig, A. Habel, H.-J. Kreowski and F. Parisi-Presicce, From graph grammars to high level replacement systems, in 4th Int. Workshop on Graph Grammars and their Application to Computer Science, Springer-Verlag. Lect. Notes Comp. Sci. 532 (1991) 269-291.

[11] H. Ehrig, A. Habel, H.-J. Kreowski and F. Parisi-Presicce, Parallelism and concurrency in high-level replacement systems. Math. Struct. Comp. Sci. 1 (1991).

[12] H. Ehrig and B. König, Deriving bisimulation congruences in the dpo approach to graph rewriting, in Foundations of Software Science and Computation Structures FoSSaCS '04, Springer. Lect. Notes Comput. Sci. 2987 (2004) 151-166.

[13] H. Ehrig, H.-J. Kreowski, U. Montanari and G. Rozenberg, editors, Handbook of Graph Grammars and Computing by Graph Transformation, Volume 3: Concurrency, Parallelism and Distribution. World Scientific (1999).

[14] H. Ehrig, M. Pfender and H.J. Schneider, Graph-grammars: an algebraic approach, in IEEE Conf. on Automata and Switching Theory (1973) 167-180.

[15] F. Gadducci and U. Montanari, A concurrent graph semantics for mobile ambients, in Mathematical Foundations of Programming Semantics MFPS '01, ENTCS. Elsevier 45 (2001).

[16] H.-J. Kreowski, Transformations of derivation sequences in graph grammars. Lect. Notes Comput. Sci. 56 (1977) 275-286.

[17] S. Lack and P. Sobociński, Adhesive categories, in Proceedings of FOSSACS '04, Springer. Lect. Notes Comput. Sci. 2987 (2004) 273-288.

[18] S. Lack and P. Sobociński, Quasitoposes, quasiadhesive categories and Artin glueing, in preparation (2005).

[19] J. Lambek and P.J. Scott, Introduction to higher order categorical logic, Cambridge studies in advanced mathematics. Cambridge University Press 7 (1986).

[20] R. Milner, Bigraphical reactive systems: Basic theory, Technical Report 523, Computer Laboratory, University of Cambridge (2001).

[21] U. Montanari, M. Pistore and F. Rossi, Modelling concurrent, mobile and coordinated systems via graph transformations, in Handbook of Graph Grammars and Computing by Graph Transformation, edited by H. Ehrig, H.-J. Kreowski, U. Montanari and G. Rozenberg, World Scientific 3 (1999) 189-268.

[22] G. Rozenberg, editor, Handbook of Graph Grammars and Computing by Graph Tranformation, Volume 1: Foundations. World Scientific (1997).

[23] V. Sassone and P. Sobociński, Deriving bisimulation congruences using 2-categories. Nordic J. Comput. 10 (2003) 163-183.

[24] V. Sassone and P. Sobociński, Congruences for contextual graph-rewriting, Technical Report RS-04-11, BRICS, University of Aarhus (June 2004). 